\title{
Emergent Kalb-Ramond fields from a dimer model
}

\author{
Luis Lozano๑* and Hugo García-Compeán ${ }^{\dagger}$ \\ Departamento de Física, Centro de Investigación y de Estudios Avanzados del IPN P.O. Box 14-740, \\ CP. 07000, México D.F., México
}

(Received 1 August 2019; published 17 September 2019)

\begin{abstract}
We discuss the emergence of a Kalb-Ramond field and string charge in the lattice and consider the local bosonic model with rotor variables placed on the faces of a cubic lattice. We give the coupling model consisting of the Maxwell fields and the Kalb-Ramond field. This construction naturally incorporates the emerging coupling between both gauge and string fields. In the process, an object that resembles a D-brane on the lattice is introduced.
\end{abstract}

DOI: 10.1103/PhysRevD.100.066015

\section{INTRODUCTION}

Recently a great deal of work has been done on the subject of the topological phases of matter and topological order (see for instance [1,2]). Moreover, it is now believed that the diverse types of matter and their interactions would originate from the existence of a system formed by quantum bits of information (see [1,3,4] and references therein). Thus, the matter can be regarded as an emergent object coming from diverse ways of organizing a few degrees of freedom in local lattice bosonic models. In this context, there is a proposal in which electrons and photons and their interaction can emerge from qubits in a string-net liquid. Thus, electrons and photons can be viewed as collective models of a string-net model [3].

Beyond the model that gives rise to electrodynamics, in turn, diverse string-net liquids can lead to different types of gauge bosons and fermions with more general properties. Thus, it is possible to find gauge bosons and fermions that behave as gluons and quarks from an appropriate string-net local qubit model. This means that QCD can be obtained as an emergent theory $[5,6]$. Later, interesting generalizations to theories of gauge fields and massless fermions in any dimension and for any gauge group were also constructed [6]. In particular, the standard model of particles can be stated in this context. However, the prediction of this description implies the existence of discrete groups, which can be interpreted as cosmic strings in a very early Universe.

\footnotetext{
*1lozano@fis.cinvestav.mx

†compean@fis.cinvestav.mx
}

Published by the American Physical Society under the terms of the Creative Commons Attribution 4.0 International license. Further distribution of this work must maintain attribution to the author(s) and the published article's title, journal citation, and DOI. Funded by SCOAP ${ }^{3}$.
On the other hand, superstring theory is a theory from which it is possible as well to incorporate gauge bosons and fermions in a very different way (see for instance [7-9]). This procedure requires the introduction of the idea of compactification. Roughly speaking, this implies making the extra dimensions compact and small in order to get the theory in four dimensions. These considerations have been problematic, and the construction of sensible field theories requires one to avoid the swampland (for a review see for instance $[10,11])$. Thus, it seems natural to search for other alternatives.

In the 1990s with the advent of string dualities and M-theory, there were some proposals involving the possible origin of the fundamental strings and their properties, as objects derived from more fundamental degrees of freedom. These degrees of freedom are the nonperturbative objects known as D0-branes. A gas of $N$ of these D0-branes is, under certain considerations, described by matrix $N \times N$ quantum mechanics known as matrix theory [12]. In AdS/CFT correspondence [13], the gravitational fields are also emergent. Gravity also can be considered an emergent interaction in matrix models of gravity [14]. For a review of different aspects of emergent gravity, see [15]. Thus, in superstring theories not only do fermions and gauge fields emerge, in closed strings, gravity arises at low energies.

The claim of deriving theories for a few degrees of freedom localized in some region of spacetime is more general in the sense that not only the diverse types of matter can be derived from the local models of qubits. The idea is that the gravitational degrees of freedom, and moreover spacetime itself, might be obtained in this way.

With the arrival of many new techniques from condensed matter physics, many efforts have been made to obtain gravitons and soft gravitons as emergent particles from lattice models [16-18], starting from a symmetric rank-2 tensor immersed on the vertices (diagonal terms) and on the 
faces of the lattices (off-diagonal terms). Higher-rank symmetric tensor generalizations of these works were also proposed in Refs. [19-21].

Thus, in the present article we propose a local bosonic model that consists of regarding the fundamental string and some of its properties, such as the Kalb-Ramond charge, as emergent objects from a local lattice model. We work with an antisymmetric rank-2 tensor (see $[9,22]$ for a review of Kalb-Ramond fields) and, in particular, we obtain emergent Kalb-Ramond fields from a lattice model. With this purpose, we first introduce the model for electromagnetism, and then we combine both models to obtain the coupling of the Kalb-Ramond field potential to the string charge and to the electric field. Earlier lattice models incorporating KalbRamond fields were proposed in [23,24]. In [23] a Higgs mechanism for the Kalb-Ramond fields is proposed by coupling them to a string that eventually condensates. Moreover, in Ref. [24] a non-Abelian tensor gauge theory is implemented in the cubic lattice through the consideration of Chan-Paton colors in each boundary link.

This article is organized as follows: In Sec. II we give some preliminary material concerning some facts about the Kalb-Ramond field, the Maxwell field, and their coupling. We also revisit the photon model and the partition function. Section III is the main part of our paper, and it is devoted to proposing our model of the emerging Kalb-Ramond field and the string charge. Moreover, in this section we also give the lattice model of the emerging coupling of the mentioned fields, which requires the introduction of the idea of a D-brane in the lattice. Finally, in Sec. IV we give our final remarks.

\section{PRELIMINARIES}

In the present section we give some preliminaries for Sec. III. Here we introduce the notation and conventions we follow in this article. We start by reviewing the field theory of the Kalb-Ramond field including its sources [21-23].

\section{A. String charge density}

We first review the string charge by introducing the antisymmetric Kalb-Ramond field potential $\mathcal{A}_{\mu \nu}=-\mathcal{A}_{\nu \mu}$ on a $(3+1)$-dimensional Minkowski spacetime and its associated field strength $\mathcal{F}_{\mu \nu \rho}$ given by

$$
\mathcal{F}_{\mu \nu \rho}=\partial_{\mu} \mathcal{A}_{\nu \rho}+\partial_{\nu} \mathcal{A}_{\rho \mu}+\partial_{\rho} \mathcal{A}_{\mu \nu}
$$

These fields have a great similarity with the Maxwell gauge field potential $A_{\mu}$ and the electromagnetic field strength $F_{\mu \nu}$ (note the ranks). In the electromagnetic theory, the electric current $j^{k}$ (one index $k=1,2,3$ ) and the electric charge density $q\left(=j^{0}\right)$ appear in

$$
\partial_{\nu} F^{\mu \nu}=j^{\mu} .
$$

For the Kalb-Ramond field strength we have

$$
\frac{1}{\kappa^{2}} \partial_{\rho} \mathcal{F}^{\mu \nu \rho}=j^{\mu \nu}
$$

where $\kappa$ is a constant, which is needed to keep the units, and $j^{\mu \nu}$ (two indices) is an antisymmetric tensor $\left(j^{\mu \nu}=-j^{\nu \mu}\right.$ ). The components $j^{0 k}=\vec{j}^{0}$ are called the Kalb-Ramond charge density or, for simplicity, the string charge density. They satisfy $\nabla \cdot \vec{j}^{0}=0$, and in the case of static strings, we have $j^{i k}=0$, which is the case we adopt in our lattice model; only $j^{0 k}$ will be nonvanishing.

For static strings, we have to consider that $\partial_{\rho} \mathcal{F}^{i k \rho}=0$, and also that

$$
\partial_{\ell} \mathcal{F}^{0 k \ell}=\kappa^{2} j^{0 k}
$$

There is a canonical conjugate variable $\Pi^{k \ell}$ to the string field potential $\mathcal{A}^{k \ell}$ which can be obtained as $\Pi^{k \ell}=\mathcal{F}^{0 k \ell}$ (see Sec. II B and [22]). We can also introduce a vector $\vec{B}_{\mathcal{F}}$ field related to the Kalb-Ramond field strength by

$$
\mathcal{F}^{0 k \ell}=\varepsilon^{k \ell m} B_{\mathcal{F} m} .
$$

It is called the field strength dual to $\mathcal{F}$, and joining these last equations we obtain

$$
\varepsilon^{k \ell m} \partial_{\ell} B_{\mathcal{F} m}=\kappa^{2} j^{0 k},
$$

which is like the Ampere's law but for the string charge densities.

\section{B. The Hamiltonian}

Now we turn to the Hamiltonian formulation of the free static Kalb-Ramond field strength (see $[9,22,23])$. First we check the Lagrangian density

$$
\mathcal{L}=-\frac{1}{6 \kappa^{2}} \mathcal{F}^{\mu \nu \rho} \mathcal{F}_{\mu \nu \rho},
$$

which is invariant under the gauge transformations $\mathcal{A}_{i j} \rightarrow$ $\mathcal{A}_{i j}+\partial_{i} f_{j}-\partial_{j} f_{i}$ since we are working in the static case. The canonical momentum conjugate to $\mathcal{A}_{i j}$ is found to be (check [22] for details)

$$
\Pi^{i j}=\dot{\mathcal{A}}^{i j}+\partial^{i} \mathcal{A}^{j 0}+\partial^{j} \mathcal{A}^{0 i} .
$$

This is a two-rank antisymmetric tensor that satisfies $\dot{\Pi}^{0 i}=\partial_{k} \Pi^{k i}$, and the weak constraints

$$
\Pi^{0 i} \approx 0, \quad \partial_{i} \Pi^{i k} \approx 0 .
$$


Thus to make the Hamiltonian density

$\mathcal{H}=\frac{1}{4} \Pi^{i j} \Pi_{i j}+\frac{1}{2} \mathcal{F}^{123} \mathcal{F}_{123}+\mathcal{A}_{j 0} \partial_{i} \Pi^{i j}-\dot{\mathcal{A}}_{0 j} \Pi^{0 j}$.

The first term is analogous to the term $E^{2}$ for electrodynamics and the second term for $B^{2}$. They can be put together in the Hamiltonian as one term like $\mathcal{F}_{i j k}^{2}$. The third and fourth terms have been added to the Hamiltonian, so we can modify them to fit the lattice model, keeping them as constraints. As can be observed from the last section, the term $\partial_{i} \Pi^{i j}$ is the string charge $-\kappa^{2} j^{0 j}$, so if there is a term with $\mathcal{A}_{j 0}$ as a coefficient, it has to be interpreted as the string charge. Depending on the gauge chosen, this term can be taken as 0 , but we leave this for later.

\section{Branes}

We present some information about D-branes, which will help us have a better understanding of how the couplings occur. In the continuous case (see [25]), the open strings are free on the space (in our case a 3-dimensional space) but couple to D-branes on their boundaries or endpoints. These objects are $\mathrm{D}$-dimensional bodies that also live free on the space; as a matter of fact, a 0-brane is a point, and a 1-brane is a string. In this way, we can observe that a 2-brane is a membrane, which is a 2-dimensional object, and so on for the following dimensions.

Objects like these can be represented on a lattice by restricting the space on which one is allowed to work inside the brane. Different examples are shown in Fig. 1. Also, it is important to have in mind how a string couples to a D-brane, as can be seen in Fig. 2, where one of the ends of a (a)

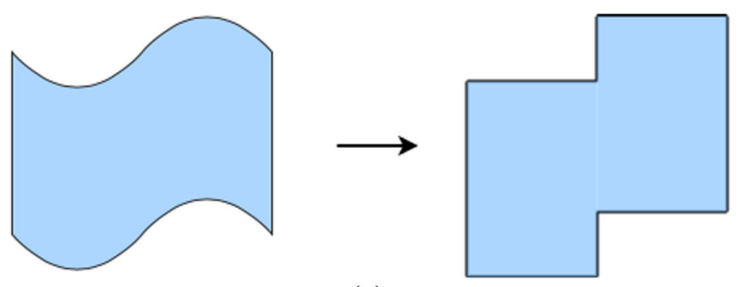

(c)

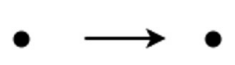

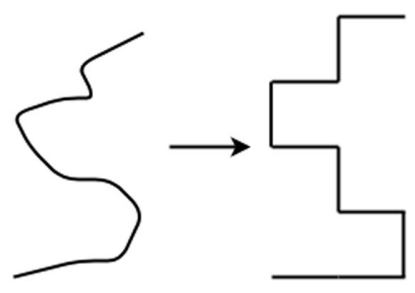

(b)
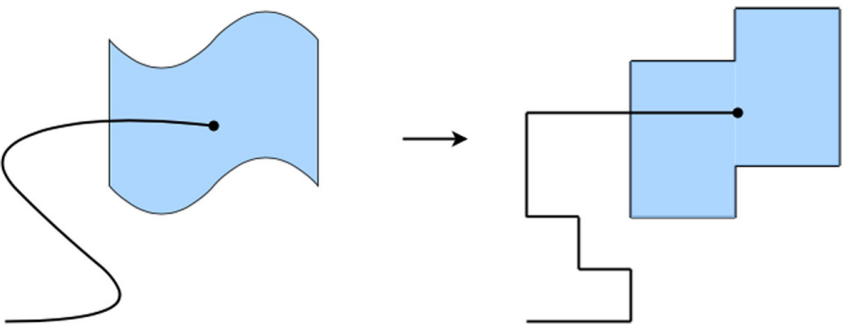

FIG. 2. Continuous and lattice strings attached to 2-branes.

string is attached to a 2-brane. Closed strings do not attach to D-branes, but open strings attach to D-branes from both sides - to the same D-brane or to a different one. For the coupling model, we consider this and restrict ourselves to the case in which the string carries Kalb-Ramond charge; only electric fields will be treated on the D-branes.

\section{Couplings}

Now we want to present the different couplings we encounter in the lattice model, beginning with the coupling between a charge and its corresponding field, with the final objective of coupling the electric field with the string field (or the Kalb-Ramond field).

First, we note the coupling between the gauge field potential $A_{\mu}$ and the electric charge density vector $j^{\mu}$, which is given by

$$
A_{\mu} j^{\mu}
$$

in the Hamiltonian density. The conservation of the electric charge density $\partial_{\mu} j^{\mu}=0$ is implied by the gauge invariance under the transformation

$$
A_{\mu}=A_{\mu}+\partial_{\mu} f
$$

as can be seen from the variation of the coupling action (see [9]). Here $f$ is an arbitrary scalar field.

For the Kalb-Ramond charge and field we have the general form of the coupling as

$$
\mathcal{A}_{\mu \nu} j^{\mu \nu}
$$

and, as in the electromagnetic case, invariance under gauge transformation

$$
\mathcal{A}_{\mu \nu} \rightarrow \mathcal{A}_{\mu \nu}+\partial_{\mu} \lambda_{\nu}-\partial_{\nu} \lambda_{\mu}
$$

where the $\lambda_{\mu}$ 's are arbitrary vector fields, implies the conservation of string charge $\partial_{\mu} j^{\mu \nu}=0$.

When the string is open, there is another type of coupling on the boundaries given between the KalbRamond field and the electric field. For this case, first we have to split the coordinates as follows: coordinates normal or perpendicular to the electric field $\left(\mu_{\perp}\right)$, and
FIG. 1. Continuous and lattice D-branes. Diagram (a) shows a 0-brane on the lattice, (b) shows a 1-brane on the lattice, and (c) shows a 2-brane. 
coordinates along the electric field or parallel to it $\left(\mu_{\|}\right)$, as $\mu=\left(\mu_{\perp}, \mu_{\|}\right)$. Following this, we also have to couple the gauge transformations as

$$
\begin{aligned}
& \mathcal{A}_{\mu \nu} \rightarrow \mathcal{A}_{\mu \nu}+\partial_{\mu} \lambda_{\nu}-\partial_{\nu} \lambda_{\mu}, \\
& A_{\mu_{\|}} \rightarrow A_{\mu_{\|}}-\lambda_{\mu_{\|}},
\end{aligned}
$$

where the term added for the Maxwell gauge transformation is equivalent to Eq. (12).

In order to keep the string action and the Maxwell action invariant, we have to include the invariant quantity

$$
-\frac{1}{4}(F+\mathcal{A})^{\mu \|^{\nu}}(F+\mathcal{A})_{\mu_{\|} \nu}
$$

in the Hamiltonian, which, by expansion, gives rise to the term

$$
-F^{0 k} \mathcal{A}_{0 k}
$$

This is the coupling between the electric field $F^{0 k}=E^{k}$, which takes the place of the string charge away from the string, and the string field potential $\mathcal{A}^{0 k}$ (see $[9,23]$ ).

It has to be clear that the string charge couples to the string field potential along the string (which is the place where the string charge exists). On the other hand, the electric field couples to the string field potential only through the D-brane to which the string is attached, as can be seen in Fig. 3. It can also be appreciated how the string charge goes along the string, and how the electric field goes on the 2-brane.

\section{E. The photon model}

Now we present a dimer model that gives rise to photons as emergent particles (there have been many other models—see for example [16,17,19,26,27] — and many generalizations to higher order symmetric tensors-see $[16,17,20,21,25])$.

Let us first introduce the quantum dimer model (QDM, see [28]) with the simplest kinetic and potential energy terms written as

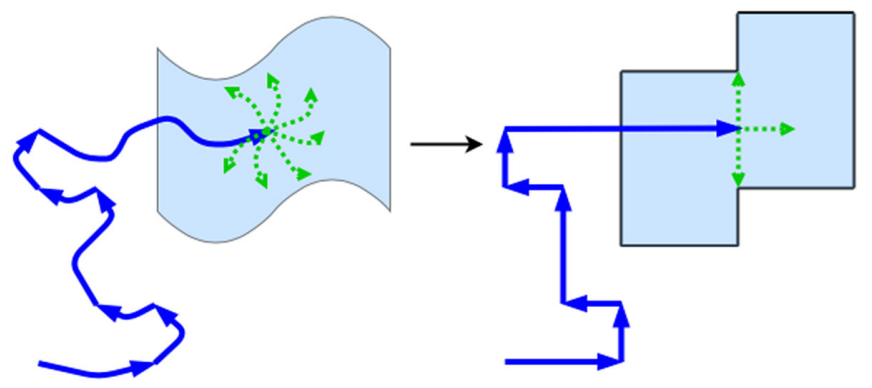

FIG. 3. Continuous and lattice string charge attached to 2-branes. The string charge is shown in blue, while the electric field is shown in green.
$H_{Q D}=\sum_{\square}\left\{-T_{1}\left(|=\rangle\langle\|||+\right.\right.$ H.c. $\left.)+T_{2}(|||\rangle\langle\||+|=\rangle\langle=|)\right\}$,

where the summation runs over all the plaquettes $(\square)$ of the lattice, in which the plaquettes are the same as the faces.

In this model, the kinetic term $T_{1}$ flips pairs of nearestneighbor parallel dimers, which are links on the lattice (see Fig. 4), and the potential term $T_{2}$ creates a repulsion between them. This model has been widely used with different purposes (see [17,20,26,29-31], but we mostly follow the meaning for the electrical part used in [17] with a slight variation in the notation as in [32].

We define on each link $(i, \alpha)\left[i=\left(i_{x}, i_{y}, i_{z}\right)\right.$ denotes the site in which the dimer begins, and $\alpha=x, y, z$ the direction to which it grows], a number operator analogous to the electric field $\hat{E}_{i \alpha}$ and its conjugate angular phase operator analogous to the potential field $\hat{A}_{i \alpha}$ (as defined in $[17,26,30,32]$ ), so these variables satisfy $\left[\hat{A}_{i \alpha}, \hat{E}_{j \beta}\right]=i \delta_{i j} \delta_{\alpha \beta}$. It is important to notice that the notation for the operators defined on the links implies that $\hat{E}_{i \alpha}=\hat{E}_{i+\hat{\alpha},-\alpha}$.

There is a constraint on the system that we have to impose on the Hilbert space because it represents the discrete form of Gauss's law for electric fields (see [16-18]),

$$
\nabla_{\alpha} \hat{E}_{i \alpha}=0,
$$

where the symbol $\nabla_{\alpha}$ means lattice differentiation or difference, and it is defined as $\nabla_{\alpha} \hat{E}_{i \beta}=\hat{E}_{i+\hat{\alpha}, \beta}-\hat{E}_{i \beta}$. Because of this constraint, the low-energy Hamiltonian has to be invariant under the gauge transformation

$$
\hat{A}_{i \alpha} \rightarrow \hat{A}_{i \alpha}+\nabla_{\alpha} f_{i}
$$

where $f_{i}$ is an arbitrary scalar field defined on the sites. This last equation is the discrete version of Eq. (12).

With all this information, the Hamiltonian for our system is given by

$$
H_{e}=\frac{K_{1}}{2} \sum_{i \alpha} \hat{E}_{i \alpha}^{2}-K_{2} \sum_{i \gamma} \cos \left(\varepsilon_{\gamma \alpha \beta} \nabla_{\alpha} \hat{A}_{i \beta}\right) .
$$

The notation employed is that of [32]. This Hamiltonian is the free 3-dimensional compact QED model (see [16]) with

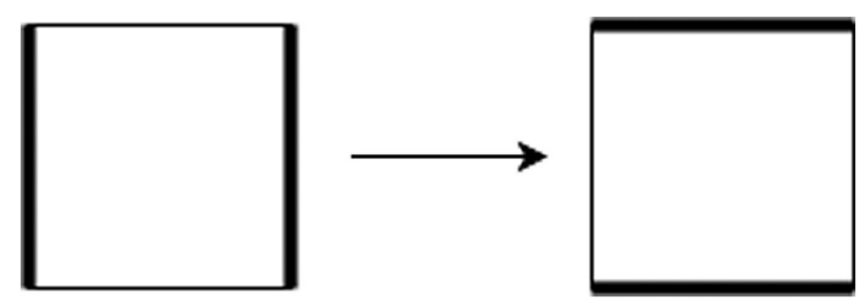

FIG. 4. The kinetic term flips the dimers as in Eq. (19). 
a deconfined photon phase (see [17,33]). The term with coefficient $K_{1}$ keeps a uniform density of dimers, and the term with coefficient $K_{2}$ flips dimers around a plaquette. The term $\varepsilon_{\gamma \alpha \beta} \nabla_{\alpha} \hat{A}_{i \beta}$ is shown in Fig. 5 (see [32]).

In order to include currents and charges in our model, we have to include a new phase angle operator $\hat{\phi}_{i}$ on the sites, and its conjugate number operator $\hat{n}_{i}$. Also, we mention an important point about Gauss's constraint: If it is violated, some defects will be generated on the sites $i$ where $\nabla_{\alpha} \hat{E}_{i \alpha} \neq 0$, and these defects carry charges of the gauge field potential $\hat{A}_{i \alpha}$ (see Fig. 6 and Ref. [17]). The way in which the defects couple to the gauge field potential is given as a term in the Hamiltonian as follows:

$$
H_{A . q}=-c_{1} \sum_{\alpha} \cos \left(\partial_{\alpha} \phi^{(q)}-A_{\alpha}\right)
$$

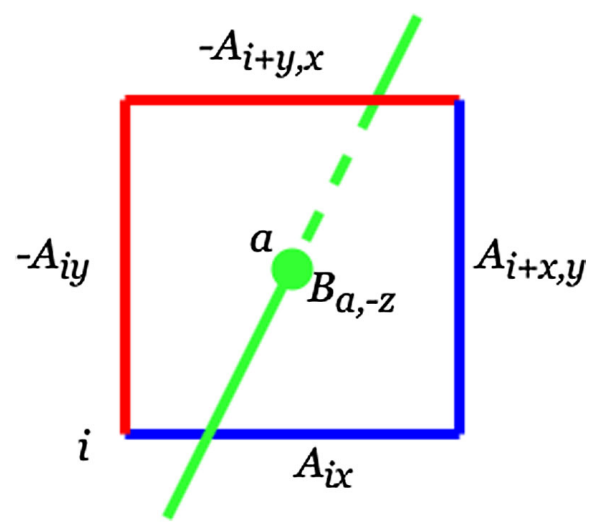

FIG. 5. The argument of the cosine function in Eq. (22) is visible, and we show how it gives rise to a dual magnetic field $-B_{a z}$. The positive field potentials are shown in blue, while the negative ones are shown in red. The magnetic field that is generated is in the opposite direction and negative, as shown in green.

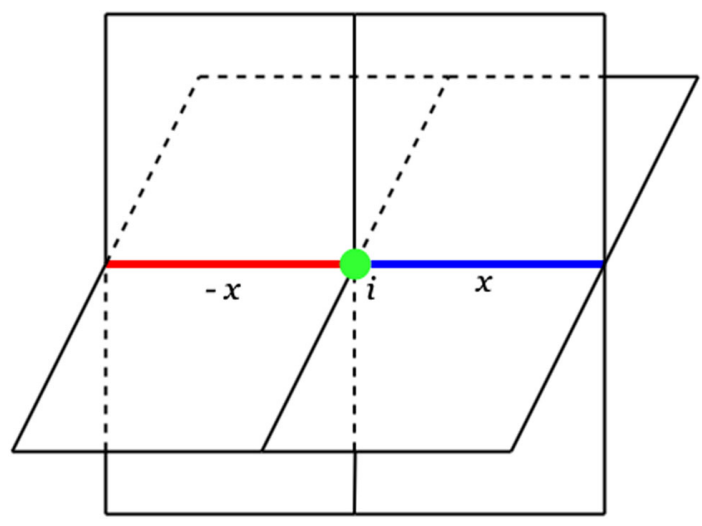

FIG. 6. A defect at site $i$ when Gauss's constraint is violated in the $\hat{x}$ direction. Again, blue is positive, red negative, and green is the charge. One can see that the 2 in the current on Eq. (28) comes from the difference of the links.
Considering the constraint (20) and the gauge transformation (21), we obtain a new Hamiltonian as

$$
\begin{aligned}
H_{\tilde{e}}= & \frac{K_{1}}{2} \sum_{i \alpha} \hat{E}_{i \alpha}^{2}-K_{2} \sum_{i \gamma} \cos \left(\varepsilon_{\gamma \alpha \beta} \nabla_{\alpha} \hat{A}_{i \beta}\right)-K_{3} \sum_{i} \hat{n}_{i} \hat{A}_{i \tau} \\
& -K_{4} \sum_{i \alpha} \cos \left(\nabla_{\alpha} \hat{\phi}_{i}-\hat{A}_{i \alpha}\right) .
\end{aligned}
$$

The term with coefficient $K_{3}$ is the coupling between the electric charge and the electric field potential. It has to be clear that this term is imposed by Gauss's law in Eq. (20), in which the term $\hat{A}_{i \tau}$ can be seen as a Lagrange multiplier defined on the vertices. The term with coefficient $K_{4}$ is the gauge transformation of Eq. (23) defined on each link. It is important to notice that for the gauge transformation inside the cosine of the $K_{4}$ term to be valid in Eq. (25), there cannot be a current or $K_{3} \rightarrow \infty$ as mentioned above, and we work with the low-energy physics.

We now follow Ref. [32], so we write the path integral representation of the partition function by inserting the eigenstates of $\hat{E}_{i \alpha}$ at small imaginary time intervals $\Delta \tau$. The cosine term of $K_{2}$ can be replaced by the Villain form approximation as

$$
\begin{aligned}
& \exp \left\{K_{2} \Delta \tau \cos \left(\varepsilon_{\gamma \alpha \beta} \nabla_{\alpha} \hat{A}_{i \beta}\right)\right\} \\
& \rightarrow \sum_{\left\{B_{a \gamma}\right\}} \exp \left\{-\frac{B_{a \gamma}^{2}}{2 K_{2} \Delta \tau}+i B_{a \gamma} \varepsilon_{\gamma \alpha \beta} \nabla_{\alpha} \hat{A}_{i \beta}\right\} .
\end{aligned}
$$

The term corresponding to $K_{4}$ is given by

$$
\begin{aligned}
& \exp \left\{K_{4} \Delta \tau \cos \left(\nabla_{\alpha} \hat{\phi}_{i}-\hat{A}_{i \alpha}\right)\right\} \\
& \rightarrow \sum_{\left\{j_{i \mu}\right\}} \exp \left\{-\frac{j_{i \mu}^{2}}{2 K_{4} \Delta \tau}+i j_{i \alpha}\left(\nabla_{\alpha} \hat{\phi}_{i}-\hat{A}_{i \alpha}\right)\right\},
\end{aligned}
$$

where we have to keep in mind that Gauss's law, along with the gauge transformation, keeps this term as 0 .

Here, $B_{a \gamma}$ is an integer dual magnetic field defined on the links $(a, \gamma)$ of the dual lattice (see Fig. 5 and Ref. [34]). The relationship between the sites of the direct lattice (with indices $i, j, \ldots$, ) and the sites of the dual lattice (with indices $a, b, \ldots$,) will not be taken into account explicitly; however, it is well understood from the example of the dual magnetic field that a link in the dual lattice represents a face in the direct lattice, and sites on the dual lattice are located at the center of the cubes on the direct lattice and vice versa.

Furthermore, we define an integer dual electromagnetic tensor $\tilde{F}_{a \mu \nu}$ on the dual lattice (as a 3-dimensional analog to the one in [32]), with its row components as $\tilde{F}_{a x \nu}=$ $\left(B_{a x}, 0, E_{i z},-E_{i y}\right), \tilde{F}_{a y \nu}=\left(B_{a y},-E_{i z}, 0, E_{i x}\right)$, and $\tilde{F}_{a z \nu}=$ $\left(B_{a z}, E_{i y},-E_{i x}, 0\right)$, where $B_{a,-z}$ is the field generated as in Fig. 5. We also define the electric current on the direct lattice as $j_{i \mu}=\left(-n_{i}, k_{i x}, k_{i y}, k_{i z}\right)$, where the $k_{i \alpha}$ can be 
written in terms of the variation of the defects $\hat{\phi}$ between the vertex $i$ and the vertex $i+\alpha$. Note that for both the dual electromagnetic tensor and the current, we use $\lambda, \mu, \nu, \rho=$ $\tau, x, y, z$ and $\alpha, \beta, \gamma, \delta=x, y, z$.

We are now ready to obtain the following partition function after working with the Villain form for the cosine terms:

$$
Z_{\tilde{e}}=\sum_{\left\{\tilde{F}_{a \mu \nu}, j_{i \mu}\right\}} \exp \left\{-\frac{e_{1}^{2}}{2} \tilde{F}_{a \mu \nu}^{2}+\frac{g_{1}}{2} j_{i \mu} A_{i \mu}\right\},
$$

restricted to

$$
\varepsilon_{\lambda \mu \nu \rho} \nabla_{\mu} \tilde{F}_{a \nu \rho}+2 j_{i \lambda}=0
$$

and

$$
\nabla_{\lambda} \tilde{F}_{a \lambda \mu}=0
$$

where the time interval is chosen to give $e_{1}^{2}=K_{1} \Delta \tau=\frac{1}{K_{2} \Delta \tau}$, and $g_{1}=K_{3} \Delta \tau=\frac{1}{K_{4} \Delta \tau}$. It is also important to observe that Eqs. (28) and (29) give rise to $\nabla_{\lambda} j_{i \lambda}=0$, where in every case we are using all the subindices without taking assumptions on the time differentiation, which can be taken as 0 for simplicity.

Notice that the phases obtained in this case can be compared to those obtained in Refs. [17,26,32], but the main difference is that we are not working with holes because our Hamiltonian does not include a term that takes it into account, which means 0 hole average, as reflected on the partition function and Gauss's constraint.

In order to give some solutions, we use the restrictions to obtain

$$
j_{i \lambda}=\varepsilon_{\lambda \mu \nu \rho} \nabla_{\mu} a_{i \nu \rho}
$$

and

$$
\tilde{F}_{a \nu \rho}=\nabla_{\nu} N_{a \rho}-2 a_{a \nu \rho} .
$$

These equations are like those of Ref. [32] but on 3 dimensions $(+1$ if the time is taken into account as evolution). We observe that the current $j_{i \lambda}$ is generated by a field on the faces of the direct lattice $a_{i \nu \rho}$, which is an integer field on the links of the dual lattice.

The important phase to consider for the photon model is when the term $g_{1} \rightarrow \infty$, we can see that either the gauge $a$ or the field potential $A$ has to be 0 , which means that there is no coupling. On the other side, the other term always gives rise to a stable phase with a maximum on the energy when Eq. (31) is 0, which implies that the creation of current is directly given by the integer $N$ variable. In Ref. [32] it is called the Higgs scalar, and it is defined on the vertices; however, in our case it is defined on the links, so it can be taken as a scalar number quantity that gives rise to the electric and magnetic fields as this Higgs quantity fluctuates. The term $g_{1}$ serves as an order parameter between these two phases as $g_{1}>0$ on the phase with no couplings, to the phases on which there are couplings and currents with $g_{1}<0$.

\section{KALB-RAMOND MODEL}

Now we turn to the model for the string charges, which has the same lattice as the model above for electromagnetism, but now we define different variables. First, we introduce the integer number operators or boson numbers as $\hat{n}_{i \alpha \beta}$ on each face $i+\frac{\hat{\alpha}}{2}+\frac{\hat{\beta}}{2}$ (with the constraint $\alpha \neq \beta$ ). Conjugate to these variables, we have the phase angle operators $\hat{\theta}_{i \alpha \beta}$, defined on the same faces, related to the boson $\left(\hat{n}_{i \alpha \beta}\right)$ creation operators by $\hat{b}_{i \alpha \beta} \propto e^{-i \hat{\theta}_{i \alpha \beta}}$ and by the commutation relations on the face $i$ by

$$
\left[\hat{n}_{i \alpha \beta}, \hat{\theta}_{i \gamma \delta}\right]=i \delta_{\alpha \gamma} \delta_{\beta \delta} .
$$

We also define an antisymmetric tensor $\hat{\Pi}_{i \alpha \beta}=$ $\varepsilon_{\alpha \beta}\left(\hat{n}_{i \alpha \beta}-\bar{n}\right)$ and its conjugate antisymmetric tensor $\hat{\mathcal{A}}_{i \alpha \beta}=\varepsilon_{\alpha \beta} \hat{\theta}_{i \alpha \beta}$, where both are defined purely on the faces because of the antisymmetry $\left(\hat{\Pi}_{\alpha \beta}=-\hat{\Pi}_{\beta \alpha}\right)$. The average density of bosons per site and face is written as $\bar{n}$.

The Hamiltonian of our system is written as follows:

$$
H_{k}=H_{t}+H_{u}+H_{0}
$$

with

$$
\begin{aligned}
& H_{t}=-t \sum_{i \alpha \beta \gamma \delta} \hat{b}_{i \alpha \beta}^{\dagger} \hat{b}_{i \gamma \delta}, \\
& H_{u}=u \sum_{\alpha \beta}\left(\hat{n}_{i \alpha \beta}-\bar{n}\right)^{2},
\end{aligned}
$$

$\left.H_{0}\right|_{(i, x)}=U\left(\hat{n}_{i+\frac{\hat{x}}{2}+\frac{\hat{z}}{2}, z x}+\hat{n}_{i+\frac{\hat{x}}{2}-\frac{\hat{z}}{2}, z x}-\hat{n}_{i+\frac{\hat{y}}{2}+\frac{\hat{y}}{2}, x y}-\hat{n}_{i+\frac{\hat{\frac{y}{2}}}{2}-\frac{\hat{y}}{2}, x y}\right)^{2}$.

The first term $H_{t}$ is a hopping term between the bosons that are on the nearest neighbor faces, the second term $H_{u}$ is a repulsive interaction between them, and the last term $\left.H_{0}\right|_{(i, x)}$ is the component along the link in the $x$ direction beginning at the site $i$ relating the bosons that are on the faces touching that link. For the term $H_{0}$, the terms along the $y$ and the $z$ direction are defined similarly (see Fig. 7 for reference).

This Hamiltonian is the lattice version of Eq. (10), with the modifications mentioned below it. The $H_{0}$ term is a local constraint like the one of electromagnetism (similar to the one exposed in $[16,17,26]$ for soft gravitons and linear gravity), which is given by 


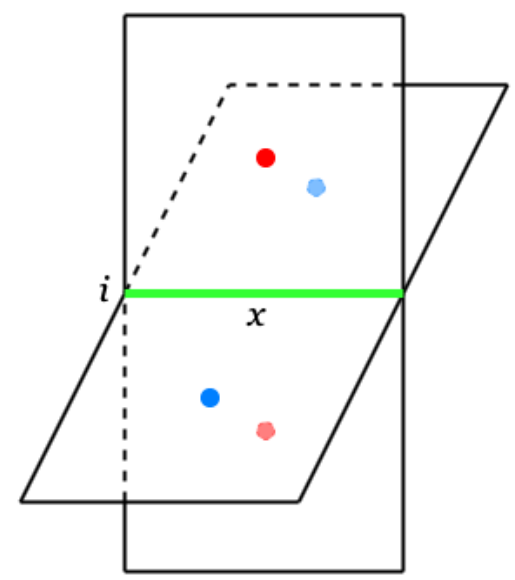

FIG. 7. The dimer $(i, x)$ (in green) is created by the interaction of the positive boson numbers (in blue) and the negative ones (in red) as the constraint $H_{0}$ dictates.

$$
\nabla_{\alpha} \Pi_{\alpha \beta}=0
$$

(with summation over the repeated index). This can take us back to the canonical momentum $\left(\Pi_{i j}\right)$ conjugate to the string field potential $\left(\mathcal{A}_{i j}\right)$ in the continuous case, which has a gauge transformation (14). In our lattice system, if we impose $U \rightarrow \infty$, the constraint has to be kept equal to 0 , and in the low-energy Hamiltonian, this constraint imposes the Hamiltonian to be invariant under a gauge transformation

$$
\hat{\mathcal{A}}_{i \alpha \beta} \rightarrow \hat{\mathcal{A}}_{i \alpha \beta}+\nabla_{\alpha} \lambda_{i \beta}-\nabla_{\beta} \lambda_{i \alpha},
$$

with the $\lambda_{i \alpha}$ 's arbitrary vector fields defined on the links.

In the low-energy physics, the $H_{t}$ term of the Hamiltonian is compactified (see $[16,17,26]$ ), and writing the $H_{u}$ with the antisymmetric tensor $\Pi_{i \alpha \beta}$, we have the following effective Hamiltonian:

$$
H_{k_{\mathrm{eff}}}=-\tilde{t} \sum_{i \alpha, \beta \neq \alpha, \gamma \neq \beta} \cos \left(K \nabla_{[\alpha} \hat{\mathcal{A}}_{i \beta \gamma]}\right)+u \sum_{i \alpha, \beta \neq \alpha} \hat{\Pi}_{i \alpha \beta}^{2}
$$

where the term inside the cosine is related to the term $\mathcal{F}^{123} \mathcal{F}_{123}$ by a constant $K$ (a modification is shown below), and the summation is carried out along the face. Here $\tilde{t}$ is a constant related to a high order perturbation of $t / U$ derived in the compactification process. Comparing our model to that of electromagnetism of the last section, we have plaquettes instead of dimers for the terms inside the cosine, as seen in Fig. 8 (see [19-21]).

If Eq. (37) is violated, we obtain the string charge (KalbRamond charge) as can be seen in the continuous case in Eqs. (4) and (8). This string charge is a defect and is taken by the string field potential $\hat{\mathcal{A}}_{i \alpha \beta}$. As can be observed from the constraints (35) and (37), and from the behavior of the string charge (6), these defects have to travel along the

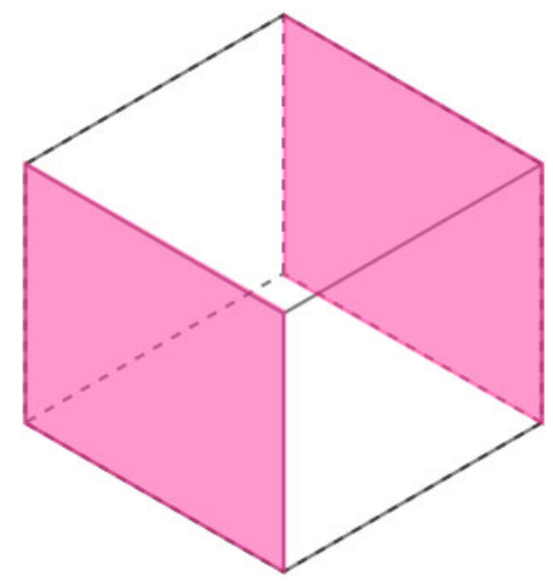

FIG. 8. The terms inside of the cosine of Eq. (39) are plaquettes instead of dimers.

links. To couple these defects to the string field in a gauge invariant way, we need to consider the gauge transformation (38) and observe that the defects can be added by arbitrary vector fields, so we have the following gauge invariant coupling term for the Hamiltonian (see [17]):

$$
H_{\mathcal{A} \cdot j}=-c_{2} \sum_{\alpha, \beta} \cos \left(\partial_{\alpha} \phi_{\beta}^{(j)}-\partial_{\beta} \phi_{\alpha}^{(j)}-\mathcal{A}_{\alpha \beta}\right),
$$

where the $\phi_{a}^{(j)}$, s are interpreted as the creation operators of the string charge, and the sum is along all the links surrounding the face $\alpha \beta$.

In order to obtain a dimer model like the electromagnetic one, we first have to define the variables required for Eq. (40). In this way, we have the boson number operator $\hat{n}_{i \alpha}$ on the link $(i \alpha)$, as well as the conjugate operator and the phase angle operator $\hat{\phi}_{i \alpha}$ defined likewise. Then, we can work with the following description:

$$
\begin{aligned}
H_{\tilde{k}}= & \frac{K_{5}}{2} \sum_{i \alpha \beta} \hat{\Pi}_{i \alpha \beta}^{2}-K_{6} \sum_{i} \cos \left(\nabla_{x} \hat{\mathcal{A}}_{i y z}\right)-K_{7} \sum_{i \alpha} \hat{n}_{i \alpha} \hat{\mathcal{A}}_{i \tau \alpha} \\
& -K_{8} \sum_{i \alpha \beta} \cos \left(\nabla_{\alpha} \hat{\phi}_{i \beta}-\nabla_{\beta} \hat{\phi}_{i \alpha}-\hat{\mathcal{A}}_{i \alpha \beta}\right) .
\end{aligned}
$$

The term with coefficient $K_{5}$ of Eq. (41) is kept the same, but the one with coefficient $K_{6}$ is modified as mentioned. The term $K_{7}$ uses $\hat{\mathcal{A}}_{i \alpha \tau}$ as a Lagrange multiplier defined on the links, and we need $K_{7} \rightarrow \infty$ in order to keep the Gausslike constraint. The last term with coefficient $K_{8}$ is the modification for the lattice of Eq. (40), implied by the $K_{7}$ restrictions.

We now take the Villain form approximation in the path integral representation for the $K_{8}$ term of Eq. (41). This will result in the following process: 


$$
\begin{aligned}
& \exp \left\{K_{8} \Delta \tau \cos \left(\nabla_{\alpha} \hat{\phi}_{i \beta}-\nabla_{\beta} \hat{\phi}_{i \alpha}-\hat{\mathcal{A}}_{i \alpha \beta}\right)\right\} \\
& \rightarrow \sum_{\left\{j_{i \mu \beta}\right\}} \exp \left\{-\frac{j_{i \mu \beta}^{2}}{2 K_{8} \Delta \tau}+i j_{i \alpha \beta}\left(\nabla_{\alpha} \hat{\phi}_{i \beta}-\nabla_{\beta} \hat{\phi}_{i \alpha}-\hat{\mathcal{A}}_{i \alpha \beta}\right)\right\},
\end{aligned}
$$

where we have to take into account that both terms will sum up to 0 and we keep the Gauss-like constraint (37) and the gauge transformation.

Now, we can proceed by defining the string field with components as $\mathcal{F}_{i \tau \alpha \beta}=\Pi_{i \alpha \beta}$ and $\mathcal{F}_{i \alpha \beta \gamma}=$ $\nabla_{\alpha} \mathcal{A}_{i \beta \gamma}+\nabla_{\beta} \mathcal{A}_{i \gamma \alpha}+\nabla_{\gamma} \mathcal{A}_{i \alpha \beta}$, where we are working with the eigenvalues of the operators. As in the electromagnetic case, we can work with the Villain form approximation for the last term of Eq. (41) to obtain the partition function (see [32])

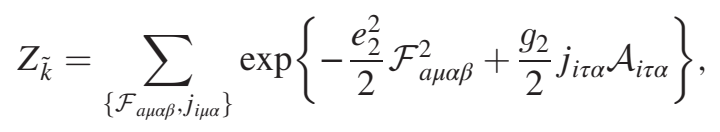

restricted to

$$
\nabla_{\alpha} \mathcal{F}_{i \mu \alpha \beta}+m j_{i \mu \beta}=0
$$

with conserved string charge. The index $\mu$ runs over $\tau, x, y$, $z$, and the other indices are only on the spatial dimensions. The term $m=2,4$ depending on how many variables create the charge (see Fig. 7). Only the $j_{i \tau \alpha}$ components survive for the string charge; the other terms appear when we have a D-brane with $D>1$ and with time evolution. The time interval is chosen to give $e_{2}^{2}=K_{5} \Delta \tau=\frac{1}{K_{6} \Delta \tau}$ and $g_{2}=K_{7} \Delta \tau=\frac{1}{K_{8} \Delta \tau}$.

We can make an analysis similar to the one in Ref. [17], but our interest is in the stable phase on the partition function of Eq. (43). We observe how the string charge $j_{i \tau \alpha}$ appears in the last step of the process as in the electromagnetic case. The spatial components of this charge are canceled by the constraint because we are working in a quasistatic case (see Ref. [9]). Also, it has to be clear that this phase is made only of closed strings formed along the links of the direct lattice, at the links where the string charge is defined.

Here we could also do the same analysis as we did for the photon model, but we observe that the pure Kalb-Ramond field can be seen as a photon model but placed on a dual lattice since all the variables defined on the links for QED are now defined on the faces. Thus, pure electrodynamics and pure antisymmetric fields are analogous or "dual." A different analysis will be obtained when working with the coupling model.

\section{A. Coupling model}

Now, we use the coupling of the Kalb-Ramond charge with the electric field, but there will be no electric charges or currents on the system; thus, we will have to modify the term $K_{4}$ of the Hamiltonian $H_{\tilde{e}}$. For this, we go back to Eqs. (15) and (16), where we can see that the coupling is through the potentials and that the defects created will be along the links. Following this, we find that the way in which these defects couple to the electromagnetic field potential is

$$
H_{A . j}=-c_{3} \sum_{\alpha_{\|}} \cos \left(\phi_{\alpha}^{(j)}+A_{\alpha}\right)
$$

where the sum is along the coordinates parallel to the direction of the electric field. With its modification to fit in the lattice, we obtain the $H_{e^{\prime}}$ for our total Hamiltonian, which is

$$
H_{e^{\prime}}=\frac{K_{1^{\prime}}}{2} \sum_{i_{b} \alpha} \hat{E}_{i_{b} \alpha}^{2}-K_{3^{\prime}} \sum_{i_{b} \alpha} \hat{n}_{i_{b} \alpha} \hat{E}_{i_{b} \alpha}
$$

where the $K_{3^{\prime}}$ is the term that couples the electric field with the string charge. The coupling term with the electric field is only along the terms parallel to the electric field, on the D-branes as

$$
H_{\mathrm{int}}=-K_{4^{\prime}} \sum_{i_{b} \alpha_{\|}} \cos \left(\hat{\phi}_{i_{b} \alpha_{\|}}+\hat{A}_{i_{b} \alpha_{\|}}\right)
$$

where the vertices $i_{b}$ are the vertices that belong to the D-branes where the electric fields are defined.

The Hamiltonian for the Kalb-Ramond charge and fields is kept exactly as in Eq. (41). Then, the total Hamiltonian will be

$$
H=H_{\tilde{k}}+H_{e^{\prime}}+H_{\mathrm{int}},
$$

and, given that we have the modification for the electric part, we will obtain a Villain form approximation in the path integral representation for the $K_{4^{\prime}}$ term as

$$
\begin{aligned}
& \exp \left\{K_{4^{\prime}} \Delta \tau \cos \left(\hat{\phi}_{i_{b} \alpha_{\|}}+\hat{A}_{i_{b} \alpha_{\|}}\right)\right\} \\
& \quad \rightarrow \sum \exp \left\{i \mathcal{A}_{i_{b} \tau \alpha_{\|}} \hat{\phi}_{i_{b} \alpha_{\|}}+i \mathcal{A}_{i_{b} \tau \alpha_{\|}} \hat{A}_{i_{b} \alpha_{\|}}\right\}
\end{aligned}
$$

Here, the defect $\hat{\phi}_{i_{b} \alpha_{\|}}$can be interpreted as the electric field, given the fact that away from the open string, the string potential couples to the electric field through a $\tau \alpha$ component. In this way, we obtain the partition function as 


$$
\begin{aligned}
Z= & \sum_{\left\{E_{i_{b} \alpha}, \mathcal{F}_{a \mu \alpha \beta}, j_{i \mu \alpha}\right\}} \exp \left\{-\frac{e_{1}^{2}}{2} E_{i_{b} \alpha_{\|}}^{2}-\frac{e_{2}^{2}}{2} \mathcal{F}_{a \mu \alpha \beta}^{2}\right. \\
& \left.+\frac{g_{1}}{2} E_{i_{b} \alpha} \mathcal{A}_{i_{b} \tau \alpha_{\|}}+\frac{g_{2}}{2} j_{i \tau \alpha} \mathcal{A}_{i \tau \alpha}\right\}
\end{aligned}
$$

restricted to

$$
\nabla_{\alpha} \mathcal{F}_{i \mu \alpha \beta}+m j_{i \mu \beta}=0
$$

and the Maxwell equations without charges [see Eqs. (28) and (29)].

We have no magnetic field here because it is a stationary case with no electric currents and charges. The string field potential couples to the electric field (on the D-brane where the electric field can be defined) and to the string charge (only along the string). In this stable phase, we have open strings attached to D-branes where electric fields are defined up to short distances (because it costs energy to keep electric fields), and we also have closed strings with only string charge. The string charge may only have a value different from 0 for the coordinates $\tau \alpha$.

Now we see that the phases on the Hamiltonian for this coupling model are diverse, but by focusing on the different locations where these couplings happen, we can obtain the stable phases. First, we get a solution from the restrictions for the electric fields as

$$
E_{i_{b} \alpha_{\|}}=\varepsilon_{\alpha_{\|} \beta \mu \nu} \nabla_{\beta} e_{i_{b} \mu \nu},
$$

where the electric field on the D-branes can be generated from fluctuations of another integer variable $b$. The current has to be conserved, and given that it travels only along the strings, we have

$$
j_{i \tau \beta}=\varepsilon_{\beta \mu \nu \rho} \nabla_{\mu} b_{i \nu \rho}
$$

where the term $b$ is an integer gauge field that gives rise to the string charge. The difference between the string charge and the electric field is where they are located, as given on the subindices. The string charge is only defined on the strings, and the electric field is only defined on the D-branes. Finally, for the restriction on Eq. (51), we obtain

$$
\mathcal{F}_{i \mu \alpha \beta}=\nabla_{\mu} N_{i \alpha \beta}-m \delta_{\tau \mu} b_{i \alpha \beta},
$$

where the term $N$ can be seen as an integer defined on the faces of the direct lattice.

We observe that along the strings $e_{1} \rightarrow \infty, g_{1}=0$, and we will be working only with the string variables. Here, the important phase is when $g_{2} \rightarrow \infty$, which is when there is no coupling, so the Kalb-Ramond field potential and its conjugate field are given by the fluctuations of the integer quantity $N$, which can be considered as the Higgs variable on the faces. At the maximum for the Hamiltonian, this term has to be 0 , so there is a balance between these fluctuations and the gauge fields $b$ that generate the string charge; however, this can only happen when the term $g_{2}$ is finite or close to 0 . When this happens, the current is created by the fluctuations of the variable $b$, which also affects the creation of the string fields.

The term $g_{2}$ can work as an order parameter that takes the system from a stable phase with pure string fields $g_{2}>0$ to a stable phase with couplings between the string charges and the gauge potential fields $g_{2}<0$.

When we take into consideration the D-branes, we have $g_{2}=0$, where the electric fields are generated, as mentioned, by the fluctuations of an integer gauge field variable $b$. Here, the string fields are only generated by the fluctuations of the Higgs term $N$ when $g_{1}$ is finite. As the term $g_{1} \rightarrow \infty$ there is no coupling; thus, the $b$ does not generate the electric field, and only the string terms are generated.

\section{FINAL REMARKS}

In the present article we have proposed a model to obtain electromagnetism $(3+1)$ dimensions from a dimer model. We have also found its corresponding partition function and the dual electromagnetic tensor in $3+1$ dimensions. The model is assumed to be quasistatic in 3 spatial dimensions, but the extensions to evolve in time have been emphasized on the model. It is also important to remark that we used, as the source for the electric and magnetic fields, the electric charges and currents instead of the field potentials (see Refs. [17,18,35]). This has to be taken into account when calculating the correlation functions.

In addition, we have obtained Kalb-Ramond fields and charges from a dimer model in $3+1$ dimensions. We again assumed that the system is quasistatic, but we have pointed out on the model how to evolve in time and the places where the field potentials grow. Its source is the string charge, and because the model is quasistatic, only the components that run along the strings $j_{i \tau \alpha}$ can be different from 0 .

In the last section, we have been able to couple both models into one $3+1$-dimensional dimer model, this time taking the string charges as the source. We have also pointed out that the coupling of the string charges with the string field potentials has to be along the strings (open or closed), while the coupling of the string field potential with the electric field has to be on the D-branes attached at the endpoints of the open strings.

In Ref. [35], the correlation functions were obtained using Monte Carlo simulations. It would be interesting to apply these methods within this context and compute some quantum observables. In the near future we would like to search for a relation to the results involving Kalb-Ramond 
fields [23,24]. Moreover, the model of the antisymmetric field studied in the present article could be coupled to the symmetric model (as in Refs. [16-18]) as a solution to the problem of finding linearized gravity as an emergent theory from lattice models. The resulting model would be interesting in the study of gravitational models with torsion [36]. We are also interested in studying the possible relationship of our results in the context of fermion-fermion duality [37]. Some of these issues will be reported elsewhere.

\section{ACKNOWLEDGMENTS}

L. L. would like to thank CONACyT for support through CVU Grant No. 594425.
[1] X. G. Wen, Quantum Field Theory of Many-Body Systems (Oxford University Press, Oxford, 2004).

[2] E. Witten, Three lectures on topological phases of matter, Riv. Nuovo Cimento 39, 313 (2016).

[3] M. A. Levin and X. G. Wen, Colloquium: Photons and electrons as emergent phenomena, Rev. Mod. Phys. 77, 871 (2005).

[4] X. G. Wen, Four revolutions in physics and the second quantum revolution: A unification of force and matter by quantum information, Int. J. Mod. Phys. B 32, 1830010 (2018).

[5] X. G. Wen, Quantum order from string net condensations and origin of light and massless fermions, Phys. Rev. D 68, 065003 (2003).

[6] M. A. Levin and X. G. Wen, String net condensation: A physical mechanism for topological phases, Phys. Rev. B 71, 045110 (2005).

[7] M. B. Green, J. H. Schwarz, and E. Witten, Superstring Theory (Cambridge University Press, Cambridge, England, 1992), Vols. 1 and 2, 25th Anniversary Edition.

[8] J. Polchinski, String Theory (Cambridge University Press, Cambridge, England, 1998), Vols. 1 and 2.

[9] B. Zwiebach, A First Course in String Theory (Cambridge University Press, Cambridge, England, 2009).

[10] E. Palti, The swampland: Introduction and review, Fortschr. Phys. 67, 1900037 (2019).

[11] M. Yamazaki, From swampland to phenomenology and back, arXiv:1904.05357.

[12] T. Banks, W. Fischler, S. H. Shenker, and L. Susskind, M theory as a matrix model: A conjecture, Phys. Rev. D 55, 5112 (1997).

[13] O. Aharony, S. S. Gubser, J. M. Maldacena, H. Ooguri, and Y. Oz, Large $\mathrm{N}$ field theories, string theory and gravity, Phys. Rep. 323, 183 (2000).

[14] P. Di Francesco, P. H. Ginsparg, and J. Zinn-Justin, 2-D gravity and random matrices, Phys. Rep. 254, 1 (1995).

[15] S. Carlip, Challenges for emergent gravity, Stud. Hist. Phil. Sci. B 46, 200 (2014).

[16] Z. C. Gu and X. G. Wen, Emergence of helicity \pm 2 modes (gravitons) from qubit models, Nucl. Phys. B863, 90 (2012).

[17] C. Xu, Algebraic liquid phase with soft graviton excitations, Phys. Rev. B 74, 224433 (2006).
[18] C. Xu and P. Horava, Emergent gravity at a Lifshitz point from a Bose liquid on the lattice, Phys. Rev. D 81, 104033 (2010).

[19] M. Pretko, Subdimensional particle structure of higher rank U(1) spin liquids, Phys. Rev. B 95, 115139 (2017).

[20] C. Xu and C. Wu, Resonating plaquette phases in SU(4) Heisenberg antiferromagnet, Phys. Rev. B 77, 134449 (2008).

[21] M. Pretko, Generalized electromagnetism of subdimensional particles: A spin liquid story, Phys. Rev. B 96, 035119 (2017).

[22] R. K. Kaul, Quantization of free field theory of massless antisymmetric tensor gauge field of second rank, Phys. Rev. D 18, 1127 (1978).

[23] S. J. Rey, The Higgs mechanism for Kalb-Ramond gauge field, Phys. Rev. D 40, 3396 (1989).

[24] S. J. Rey and F. Sugino, A nonperturbative proposal for nonabelian tensor gauge theory and dynamical quantum Yang-Baxter maps, arXiv:1002.4636.

[25] D. Bulmash and M. Barkeshli, The Higgs mechanism in higher-rank symmetric $U(1)$ gauge theories, Phys. Rev. B 97, 235112 (2018).

[26] L. Balents, L. Bartosch, A. Burkov, S. Sachdev, and K. Sengupta, Putting competing orders in their place near the Mott transition. II. The doped quantum dimer model, Phys. Rev. B 71, 144509 (2005).

[27] M. Levin and X. G. Wen, Fermions, strings, and gauge fields in lattice spin models, Phys. Rev. B 67, 245316 (2003).

[28] D. S. Rokhsar and S. A. Kivelson, Superconductivity and the Quantum Hard-Core Dimer Gas, Phys. Rev. Lett. 61, 2376 (1988).

[29] R. Moessner, S. L. Sondhi, and E. Fradkin, Short-ranged resonating valence bonds physics, quantum dimer models and Ising gauge theories, Phys. Rev. B 65, 024504 (2001).

[30] E. H. Fradkin and S. Kivelson, Short range resonating valence bond theories and superconductivity, Mod. Phys. Lett. B 04, 225 (1990).

[31] E. Fradkin, D. A. Huse, R. Moessner, V. Oganesyan, and S.L. Sondhi, On bipartite Rokhsar-Kivelson points and Cantor deconfinement, Phys. Rev. B 69, 224415 (2004). 
[32] S. Sachdev and M. Vojta, Translational symmetry breaking in two-dimensional antiferromagnets and superconductors, J. Phys. Soc. Jpn. Suppl B 69, 1 (2000).

[33] A. M. Polyakov, Quark confinement and topology of gauge groups, Nucl. Phys. B120, 429 (1977).

[34] E. H. Fradkin and L. Susskind, Order and disorder in gauge systems and magnets, Phys. Rev. D 17, 2637 (1978).
[35] D. A. Huse, W. Krauth, R. Moessner, and S. L. Sondhi, Coulomb and Liquid Dimer Models in Three Dimensions, Phys. Rev. Lett. 91, 167004 (2003).

[36] R. T. Hammond, Torsion gravity, Rep. Prog. Phys. 65, 599 (2002).

[37] G. Palumbo, Fermion-fermion duality in $3+1$ dimensions, arXiv:1907.01501. 